\title{
A LINEAR-QUADRATIC STABILIZATION SYSTEM FOR A CANARD-CONTROLLED MISSILE
}

\author{
W. Bużantowicz ${ }^{*}$
}

\begin{abstract}
This paper examines a linear-quadratic stabilization system for a canard-controlled missile. An analytical method for selecting the weighting elements of the gain matrix in the feedback loop is also proposed, eliminating the need for an iterative solution to the Riccati equation. The proposed solution was evaluated using simulation tests.
\end{abstract}

Keywords: anti-aircraft missile, autopilot, linear-quadratic regulator

\section{Introduction}

The nonstationary nature of the missile airframe determines the changes in the characteristics of the entire guidance loop. In extreme cases, this may compromise its stability. To compensate for this highly undesirable effect, a stabilizing device is installed onboard the missile. Control can be provided by any regulator that is able to track the set trajectory to a specified level of accuracy. In this paper, a linear-quadratic regulator $(\mathrm{LQR})$ will be considered for the performance of this function.

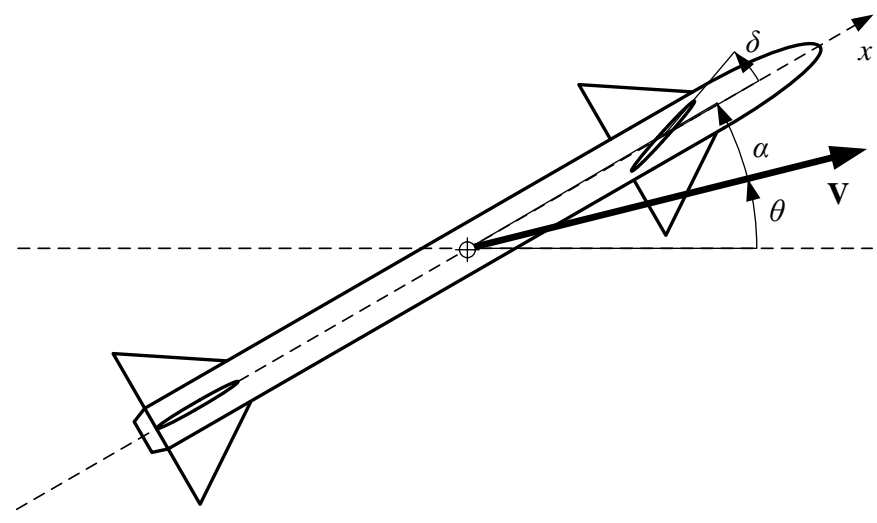

Fig. 1: Canard-controlled missile configuration

For linear time-invariant (LTI) systems, the weighting coefficients of an LQR can be easily determined by means of an algorithm. The problem becomes more complex when the analyzed system contains nonlinear and nonstationary features. In such cases, when solving the Riccati equation to find the coefficients of the gain matrix in the feedback loop, numerical computation methods are commonly applied to a set of static coefficients describing the state of the system in a finite time horizon. The main difficulty with this approach is the requirement for computations to be carried out in real time (Çimen, 2008). There are also certain complications related to the application of numerical methods, such as the bad conditioning of the matrices.

This paper proposes an analytical method for selecting the weighting elements of the gain matrix, eliminating the need for an iterative solution to the Riccati equation. The method is applied to a cruciform canard-controlled, roll-stabilized missile (Fig. 1). The motion of this type of missile can be separated into two perpendicular channels, allowing the control problem to be treated as planar in each.

Witold Bużantowicz, PhD Eng.: Faculty of Mechatronics and Aerospace, Military University of Technology, 2 Urbanowicza Street; 00-908, Warszawa; PL, wbuzantowicz@wat.edu.pl 


\section{Airframe dynamics model}

For controller design purposes, the dynamics of the airframe in the control plane can be approximated by the linearized vector-matrix system of equations

$$
\dot{\mathbf{x}}=\mathbf{A x}+\mathbf{B} \delta, \quad y=\mathbf{C x}+D \delta,
$$

where

$$
\mathbf{x}=\left[\begin{array}{l}
\alpha \\
\omega
\end{array}\right], \quad \mathbf{A}=\left[\begin{array}{cc}
-a_{1} & 1 \\
-a_{2} & -a_{3}
\end{array}\right], \quad \mathbf{B}=\left[\begin{array}{l}
0 \\
b
\end{array}\right], \quad \mathbf{C}=\left[\begin{array}{ll}
0 & 1
\end{array}\right], \quad D=0 .
$$

In expressions (1)-(2), the symbols have the following meaning: $\alpha$ is the angle of attack, $\omega$ is the angular rate of the airframe, and $\delta$ is the canard deflection angle. The entries of matrices $\mathbf{A}$ and $\mathbf{B}$ have the following forms:

$$
a_{1}=\frac{\rho V}{2 m} S c_{L}, \quad a_{2}=\frac{\rho V^{2}}{2 I} S l c_{M}^{\alpha}, \quad a_{3}=\frac{\rho V}{2 I} S l^{2} c_{M}^{\omega}, \quad b=\frac{\rho V^{2}}{2 I} S l c_{M}^{\delta},
$$

in which $\rho$ is the air density, $V$ is the missile velocity, $m$ is the missile mass, $I$ is the missile moment of inertia, $S$ is the characteristic surface, $l$ is the characteristic linear dimension, $c_{L}$ is the aerodynamic coefficient of the lift force, and $c_{M}^{\omega}, c_{M}^{\alpha}$ and $c_{M}^{\delta}$ are coefficients of the aerodynamic moments related to the damping, the airframe, and the canards, respectively.

\section{LQR controller design}

For the LQR, the controller settings are determined based on a quadratic cost function in the form

$$
J=\int_{0}^{\infty}\left(\mathbf{x}^{\mathrm{T}} \mathbf{Q} \mathbf{x}+\mathbf{u}^{\mathrm{T}} \mathbf{R} \mathbf{u}\right) \mathrm{d} t
$$

where $\mathbf{Q}=\mathbf{Q}^{\mathrm{T}}$ and $\mathbf{R}=\mathbf{R}^{\mathrm{T}}$ are weighting parameters for the state vector $\mathbf{x}$ and control vector $\mathbf{u}$. To simplify the consideration further, the inertia of the canard actuator is ignored; i.e., it is assumed that $u=\delta$. To determine the gain matrix $\mathbf{K}$ of the feedback loop, it is necessary to find the matrix $\mathbf{P}$ satisfying the Riccati equation

$$
\mathbf{A}^{\mathrm{T}} \mathbf{P}+\mathbf{P A}-\mathbf{P B R}^{-1} \mathbf{B}^{\mathrm{T}} \mathbf{P}+\mathbf{Q}=\mathbf{0},
$$

which in general cannot be done analytically (Çimen, 2008). However, in certain situations the solution can be obtained, providing adaptable feedback from the time-variable state of the system.

Let us assume the matrices $\mathbf{P}, \mathbf{Q}$ and $\mathbf{R}$ to be

$$
\mathbf{P}=\left[\begin{array}{ll}
p_{11} & p_{12} \\
p_{21} & p_{22}
\end{array}\right], \quad \mathbf{Q}=\left[\begin{array}{cc}
q_{1} & 0 \\
0 & q_{2}
\end{array}\right], \quad \mathbf{R}=[1],
$$

where $q_{1}$ and $q_{2}$ are positive-defined constants (Erdem and Alleyne, 2004), hence the gain matrix $\mathbf{K}$ is equal to

$$
\mathbf{K}=\mathbf{R}^{-1} \mathbf{B}^{\mathrm{T}} \mathbf{P}=\left[\begin{array}{ll}
b p_{21} & b p_{22}
\end{array}\right] .
$$

The expanded form of Eq. (5) takes the form

$$
\begin{gathered}
{\left[\begin{array}{cc}
-a_{1} p_{11}-a_{2} p_{21} & -a_{1} p_{12}-a_{2} p_{22} \\
p_{11}-a_{3} p_{21} & p_{12}-a_{3} p_{22}
\end{array}\right]+\left[\begin{array}{cc}
-a_{1} p_{11}-a_{2} p_{12} & p_{11}-a_{3} p_{12} \\
-a_{1} p_{21}-a_{2} p_{22} & p_{21}-a_{3} p_{22}
\end{array}\right]+} \\
-b^{2}\left[\begin{array}{cc}
p_{12} p_{21} & p_{12} p_{22} \\
p_{21} p_{22} & p_{22} p_{22}
\end{array}\right]+\left[\begin{array}{cc}
q_{1} & 0 \\
0 & q_{2}
\end{array}\right]=\left[\begin{array}{cc}
0 & 0 \\
0 & 0
\end{array}\right]
\end{gathered}
$$

By summation of the matrices in Eq. (8), comparison of the corresponding terms and rearrangement, noting moreover that $p_{12} \equiv p_{21}$, the following system of equations is obtained:

$$
\left\{\begin{array}{l}
-2 a_{1} p_{11}-2 a_{2} p_{21}+q_{1}=b^{2} p_{21} p_{21} \\
p_{11}-\left(a_{1}+a_{3}\right) p_{21}-a_{2} p_{22}=b^{2} p_{21} p_{22} \\
2 p_{21}-2 a_{3} p_{22}+q_{2}=b^{2} p_{22} p_{22}
\end{array}\right.
$$


Multiplying both sides of the second equation in (9) by 2 and summing the equations in (9), we have

$$
\left(1-a_{1}\right) p_{11}+\left(1-a_{1}-a_{2}-a_{3}\right) p_{21}+\left(-a_{2}-a_{3}\right) p_{22}=b^{2}\left(p_{21}+p_{22}\right)^{2}-q_{1}-q_{2} .
$$

Values of $q_{1}$ and $q_{2}$ can be freely chosen. Let assume that

$$
\left(p_{21}+p_{22}\right)^{2}-\frac{\varsigma^{2}}{b^{2}}=0 \quad \text { where } \quad \varsigma^{2}=q_{1}+q_{2} .
$$

The solutions for Eq. (11) are:

$$
p_{21}=\frac{\varsigma}{b}-p_{22} \quad \text { and } \quad p_{21}=-\frac{\varsigma}{b}-p_{22} .
$$

Using Eq. (12) to solve the system of equations (9), by Eq. (7), eight pairs of entries for the matrix $\mathbf{K}$ can be obtained. Three of them give unstable solutions (with positive feedback), and four other lead to inappropriate quality of the system response, cf. Fig. 2. These solutions should be rejected. Based on analysis of the values of the angular rate $\omega$ obtained as step responses of the airframe to commanded fin deflections, in our further considerations the gain matrix $\mathbf{K}$ will be taken to have the form

$$
\mathbf{K}=\left[\begin{array}{c}
-\frac{1}{b\left(1-2 a_{1}\right)}\left[a_{1}\left(a_{1}-a_{2}+a_{3}-b\right)+a_{2}+\sqrt{\Upsilon}\right] \\
-\varsigma+\frac{1}{b\left(1-2 a_{1}\right)}\left[a_{1}\left(a_{1}-a_{2}+a_{3}-b\right)+a_{2}+\sqrt{\Upsilon}\right]
\end{array}\right]^{\mathrm{T}}
$$

where

$$
\begin{gathered}
\Upsilon=\left(a_{1} b-a_{2}\right)^{2}+\left[a_{1}\left(a_{1}+a_{3}\right)-a_{1} a_{2}\right]^{2}-2\left(a_{1}-a_{2}+a_{3}\right)\left(a_{1}^{2} b-a_{1} a_{2}\right)+ \\
+\left(\frac{1}{2}-a_{1}\right)\left(b^{2}+4 a_{1} a_{2} b\right)
\end{gathered}
$$

and $\varsigma$ is a constant coefficient chosen experimentally.

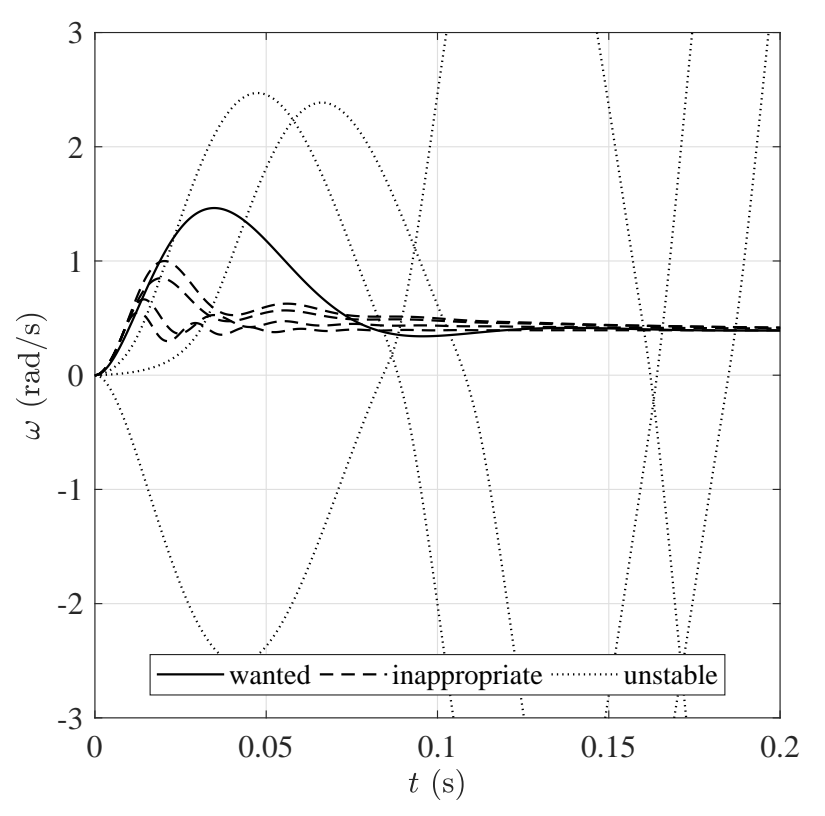

Fig. 2: Step responses of the system

Now, the control law is defined as

$$
u_{L Q R}=\left(N_{u}+\mathbf{K} \mathbf{N}_{x}\right) u-\mathbf{K} \mathbf{x}
$$

where

$$
\left[\begin{array}{l}
\mathbf{N}_{x} \\
N_{u}
\end{array}\right]=\mathbf{\Omega}^{-1}\left[\begin{array}{l}
\mathbf{0} \\
1
\end{array}\right]
$$

and

$$
\boldsymbol{\Omega}=\left[\begin{array}{ll}
\mathbf{A} & \mathbf{B} \\
\mathbf{C} & D
\end{array}\right]
$$

\section{Results and discussion}

Fig. 3 gives the selected step responses of the airframe obtained from a simulation test with the exemplary coefficients $a_{1}=5 \mathrm{~s}^{-1}, a_{2}=2350 \mathrm{~s}^{-2}, a_{3}$ $=10 \mathrm{~s}^{-1}, b=420 \mathrm{~s}^{-2}, \varsigma=0.96$ and for flight velocity $V=900 \mathrm{~m} / \mathrm{s}$. It is assumed that the missile has no thrust during the tests. The following parameters were assumed in the simulations: $m=100 \mathrm{~kg}, I=$ $35 \mathrm{~kg} \cdot \mathrm{m}^{2}, S=0.67 \mathrm{~m}^{2}, l=1.36 \mathrm{~m},|\delta| \leq 0.35$ $\mathrm{rad}$. The values of geometrical and mass coefficients and of aerodynamic forces and moments required for the Eqs. (3) were determined analytically (Kurow and Dołżanski, 1964). The value of the parameter $\varsigma$ was chosen numerically (cf. Fig. 4). The fourth-order Runge-Kutta numerical integration method was used for the derivation of approximating differential equations for the elements of the studied systems. The simulation time was $t_{s}=1 \mathrm{~s}$. 
The use of the proposed stabilization system with an LQR algorithm in the autopilot of an anti-aircraft missile would improve the quality of control by stabilizing the angular rate of the airframe. The system's transitional processes would become shorter and smoother, leading to better operating conditions for the seeker installed in the missile and more effective guidance towards an aerial target. Clearly, the approach gives rise to certain technical and implementation problems. However, discussion of these issues is beyond the scope of this work.
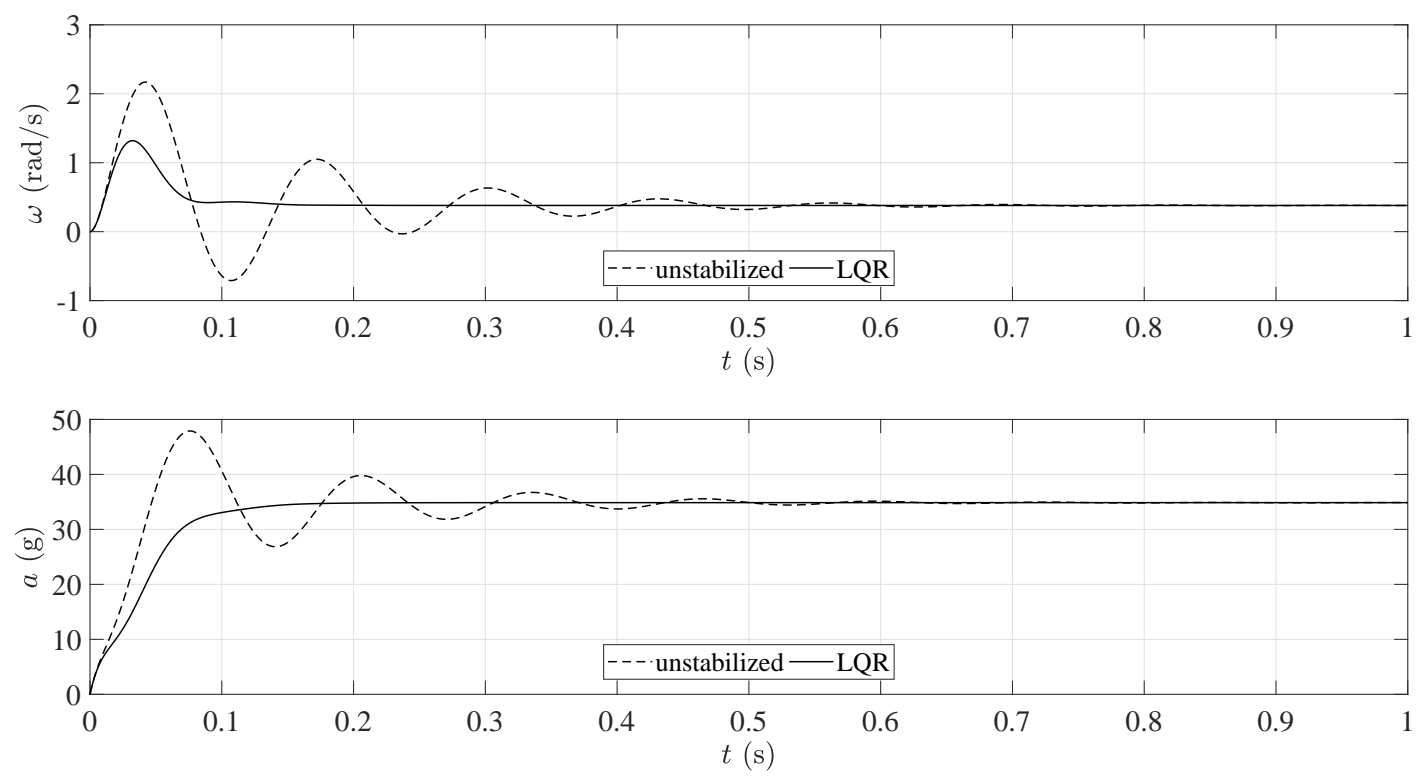

Fig. 3: Angular rates (up) and normal accelerations (below) of the airframe
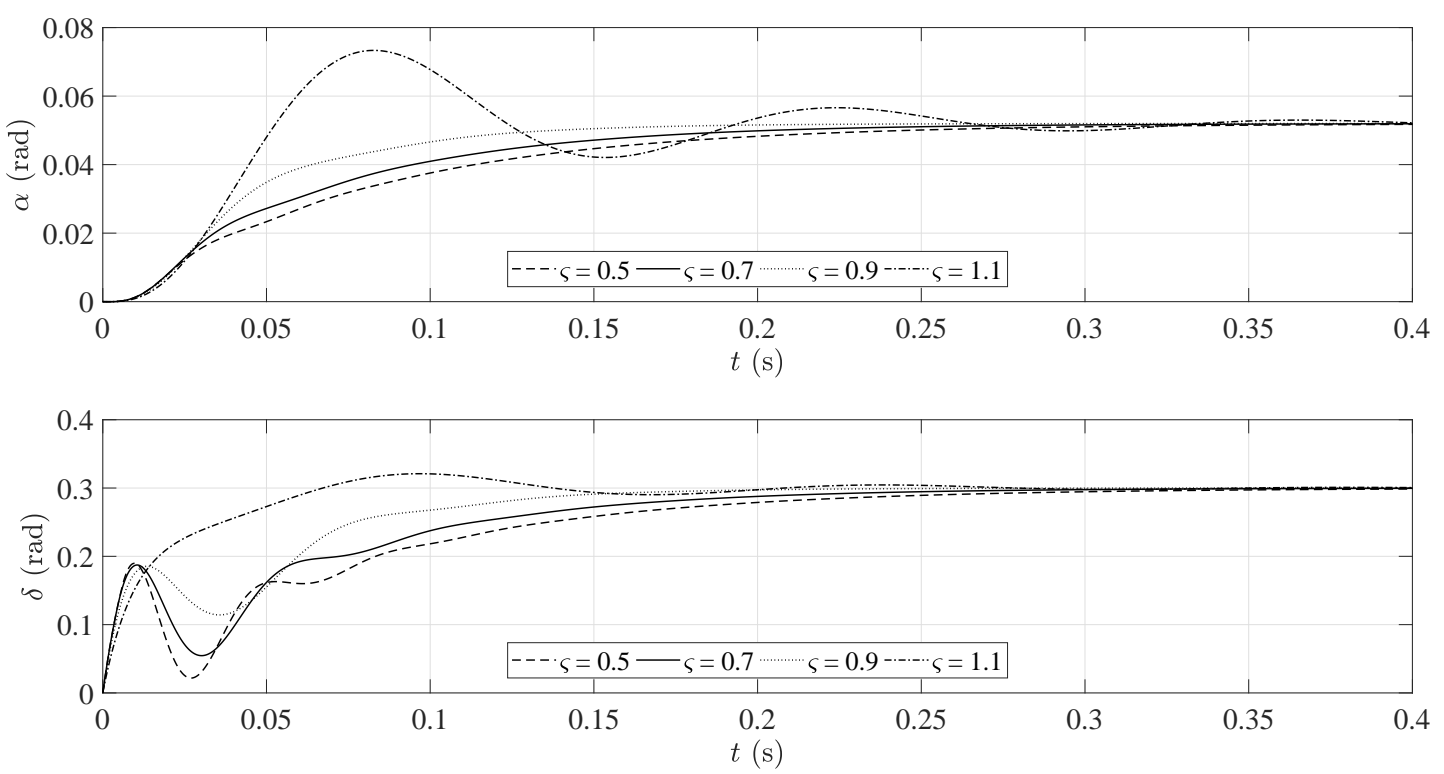

Fig. 4: Angle of attack (up) and canard deflection (below) histories for exemplary values of $\varsigma$

\section{References}

Çimen, T. (2008) State-Dependent Riccati Equation (SDRE) Control: A Survey. In: Proc. of the 17th IFAC World Congress, 41, 2, pp. 3761-3775.

Erdem, E.B. and Alleyne, A.G. (2004) Design of a class of nonlinear controllers via state dependent Riccati equations. IEEE Transactions on Control Systems Technology, 12, 1, pp. 2986-2991.

Kurow, W. and Dołżanski, J. (1964) Principles Behind the Solid-Propellant Rocket Design (in Polish). Publishing House of Ministry of National Defense, Warsaw. 